\title{
Developing and Implementing a Digital Navigation Program to Improve Outcomes for Medicare Bundle Patients Undergoing Joint Replacement Surgery
}

Jason Rogers; Farah Fasihuddin; Morgan Black; Rebecca Kann; Shelly Mei; Jonathan McLaughlin; Ashish Atreja

Icahn School of Medicine at Mount Sinai, New York, NY, United States

\section{Corresponding Author:}

Jason Rogers

Icahn School of Medicine at Mount Sinai

1 Gustave Levy Pl

New York, NY

United States

Phone: 2122416500

Email: jason.rogers@mountsinai.org

\section{Abstract}

Background: Evidence-based patient education and consistent, timely communication is key to ensuring good outcomes among joint replacement patients. Mount Sinai Hospital (MSH) participates in the mandatory CMS bundle for comprehensive joint replacement (CJR). MSH's bundled payment strategy focuses on the development of a standardized model of care, built around evidence-based best practices to achieve the triple aims of strengthening population health while controlling cost and improving the quality of care. MSH launched a comprehensive digital navigation program (DNP) to guide joint replacement patients and their caregivers through pre-surgical preparation and recovery.

Objective: The objective was to improve the quality of care for joint replacement patients through creation of a digital navigation program specifically tailored to Medicare patients (age 65+) across the continuum of care.

Methods: Mount Sinai App Lab, in collaboration with the Department of Orthopedics, developed three digital therapeutic modules that were delivered through the RxUniverse Digital Medicine platform (Rx.Health, NY). These automated messages, programmed to send at specific times, included exercise instructions, medication reminders, and suggestions for how to prepare the home for optimal recovery. Messages specifically targeted key patient outcomes: length of stay, readmissions, ambulation on postoperative day 0, and discharge disposition. Staff "prescribed" each module to patients to ensure that engagement aligned with their process of care.

Results: Clinicians, patients, and their caregivers were receptive to the DNP. Patients showed a high rate of engagement, clicking links to educational content 873 times, and patients called their case manager or surgeon's office to clarify their next steps when prompted. After 9 months, clinical outcomes for the digital navigation program were compared to other Medicare patients who had not received it. DNP patients had significantly shorter length of stay than their peers (2.81 vs 4.31 days). They also had a lower readmission rate $(1.9 \%$ vs $2.9 \%)$ as well as a higher rate of discharge to home (87.8\% vs $64.3 \%)$ and were more likely to ambulate on the day of surgery (47.9\% vs $33.3 \%$ ). Twenty patients responded to an end-of-program survey about their experience; 18 patients $(90 \%)$ agreed that the program was helpful with the process of their total joint replacement surgery, 2 patients neither agreed nor disagreed (10\%), and 0 patients disagreed. When asked about their satisfaction with the message volume, 19 patients answered "yes, this was the perfect number," (95\%) and 1 patient answered "no, I want fewer messages" (5\%). Patient qualitative feedback was very positive. Patients reported, "Texts reassured me and helped me along with my progress and recovery," and "Good support. Thank you." A third said, "The texting program serves as a great reminder as what to do and when."

Conclusions: The CJR DNP provided a direct, automated channel to educate and support patients at each stage of care. It demonstrated that digital navigation technology can be used even among non-digital native populations. It resulted in a significantly reduced length of stay and hospital readmissions among participating patients. Next steps include scaling the program across the health system and adding Spanish-language support. Similar programs are now being implemented for other surgical and disease use cases.

(iproc 2019;5(1):e15197) doi: 10.2196/15197 


\section{KEYWORDS}

mHealth; mobile health (mHealth); patient empowerment; patient monitoring

Edited by R Palacholla; this is a non-peer-reviewed article. Submitted 27.06.19; accepted 14.08.19; published 02.10.19.

Please cite as:

Rogers J, Fasihuddin F, Black M, Kann R, Mei S, McLaughlin J, Atreja A

Developing and Implementing a Digital Navigation Program to Improve Outcomes for Medicare Bundle Patients Undergoing Joint Replacement Surgery

iproc 2019;5(1):e15197

URL: http://www.iproc.org/2019/1/e15197/

doi: $\underline{10.2196 / 15197}$

PMID:

CJason Rogers, Farah Fasihuddin, Morgan Black, Rebecca Kann, Shelly Mei, Jonathan McLaughlin, Ashish Atreja. Originally published in Iproceedings (http://www.iproc.org), 02.10.2019 This is an open-access article distributed under the terms of the Creative Commons Attribution License (https://creativecommons.org/licenses/by/4.0/), which permits unrestricted use, distribution, and reproduction in any medium, provided the original work, first published in Iproceedings, is properly cited. The complete bibliographic information, a link to the original publication on http:/www.iproc.org/, as well as this copyright and license information must be included. 